\title{
Comparison among four psychophysical procedures used in lateralization*
}

\author{
WILLIAM A. YOST, ROBERT TURNER, and BYRON BERGERT \\ Communication Sciences Laboratory, University of Florida, Gainesville, Florida 32611
}

\begin{abstract}
Psychometric functions were obtained from two listeners in four psychophysical tasks. The tasks were lateralization procedures in which Os were asked to make discriminations of interaural temporal differences of a $250-\mathrm{Hz}$ tone. The four tasks were: a single-interval yes-no task, a single-interval left-right task, a two-alternative forced-choice task, and a two-interval same-different task. The theory of signal detection provides predictions relating the performances obtained in these four procedures. These predictions could not be verified in this experiment when it was assumed that the Os were listening to changes in lateral position produced by the interaural temporal difference. The data were, however, consistent with the assumption that Os use lateral motion as a cue for detection in two-interval tasks and lateral position as a cue in single-interval tasks.
\end{abstract}

Many different psychophysical procedures have been used to study lateralization of acoustical stimuli. Most of these procedures are similar to or identical to those used in other psychoacoustical research. When the stimuli are sinusoids and noise, and when Os are asked to detect the presence of the noise-masked sinusoids, the theory of signal detection (TSD) has provided a good account of the data obtained from a variety of psychophysical tasks (see Robinson \& Watson, 1972). That is, if the criterion-free measure of performance, $d^{\prime}$, is used, then there is a predictable relationship among the performances measured in a variety of paradigms.

Although the predictions of TSD have been applied to masking experiments, these predictions have seldom been applied to data obtained from investigations of lateralization. In the present study, performance in four lateralization tasks was compared. Figure 1 shows a diagram of these four procedures. In the yes-no task (Y-N), a $250-\mathrm{Hz}$ tone is presented with either no interaural difference $(\Delta T=0)$ or an interaural temporal difference presented to the right ear $(\Delta T=\tau)$ on each trial; the $O$ indicates whether or not the interaural temporal difference was presented. In the left-right task (L-R), the interaural temporal difference is added either to the left ear $\left(\Delta \mathrm{T}_{\mathrm{L}}=\tau / 2\right)$ or to the right ear $\left(\Delta \mathrm{T}_{\mathrm{R}}=\right.$ $\tau / 2)$ on each trial, and the $O$ indicates which ear received the temporal difference. ${ }^{1}$ The same-different task (S-D) is a two-interval task; in the first interval, the $250-\mathrm{Hz}$ tone is always presented with no interaural difference $(\Delta T=0)$, and either this tone or one containing the interaural temporal difference $(\Delta \mathrm{T}=\tau)$ is presented in the second interval. The $O$ indicates whether the tones presented in the two intervals were the same or different. In the two-alternative forced-choice task (2AFC), either the tone containing the interaural

*This research was supported by a National Institutes of Health training grant and Program Project grant. We thank Edward Carterette for his helpful comments. temporal difference $(\Delta \mathrm{T}=\tau)$ or the one containing no interaural difference $(\Delta \mathrm{T}=0)$ is presented in the first interval, and the other tone is presented in the second interval. The $\mathrm{O}$ indicates which interval contained the tone with the interaural temporal difference. If one assumes that in each task there are two events (two values of interaural delay) which the $\mathrm{O}$ is to discriminate between, then TSD provides predictions regarding the performance measured in the four tasks. That is, the value of $d^{\prime}$ obtained in the two single-interval tasks (L-R, Y-N) should be the same, given the same values of the interaural temporal difference. In addition, d' obtained in the S-D task should be equal to or smaller than that obtained in the single-interval tasks (see Sorkin, 1964, for a derivation of this relation). Finally, the value of $\mathrm{d}^{\prime}$ obtained in the $2 \mathrm{AFC}$ task should equal $\sqrt{2}$ times the value of $\mathrm{d}^{\prime}$ obtained in the single-interval tasks (see Green \& Swets, 1966, Chapter III, for this derivation). Thus, the present study was designed to see if these relations among various values of $\mathrm{d}^{\prime}$ obtained in these four tasks would be verified in tasks involving lateral discrimination.

\section{METHOD}

A $300-\mathrm{msec} 250-\mathrm{Hz}$ tone with a $20-\mathrm{msec}$ rise-decay time was presented to both ears of the two Os. The level of the tone was $70 \mathrm{~dB}$ SPL. An Ad-Yu delay line (Model $801 \mathrm{f}$ ) was used to present two values of interaural temporal difference $(\Delta T)$ in each procedure. Figure 1 shows the timing sequence used in each of the four procedures. In the $\mathrm{Y}-\mathrm{N}, \mathrm{S}-\mathrm{D}$, and $2 \mathrm{AFC}$ tasks, $\Delta \mathrm{T}$ was added to the right ear. In the L-R task, the interaural delay was added either to the left or to the right ear. No other interaural differences were measured at the input to the TDH-39 headphones.

$\mathbf{P}(\mathrm{C})_{2}$ (McFadden, 1970) and $\mathrm{d}^{\prime}$ were obtained in each of the four procedures and at each value of $\Delta T$. Five 100 -trial blocks of data were obtained for each $O$ in each procedure and for both values of $\Delta T$. There were, therefore, two-point psychometric functions (500 trials per point) for each procedure and for both Os. The psychometric functions related either $P(C)$, or $d^{\prime}$ to the values of $\Delta \mathrm{T}$. The a priori probability for the stimuli in each procedure was one-half. 


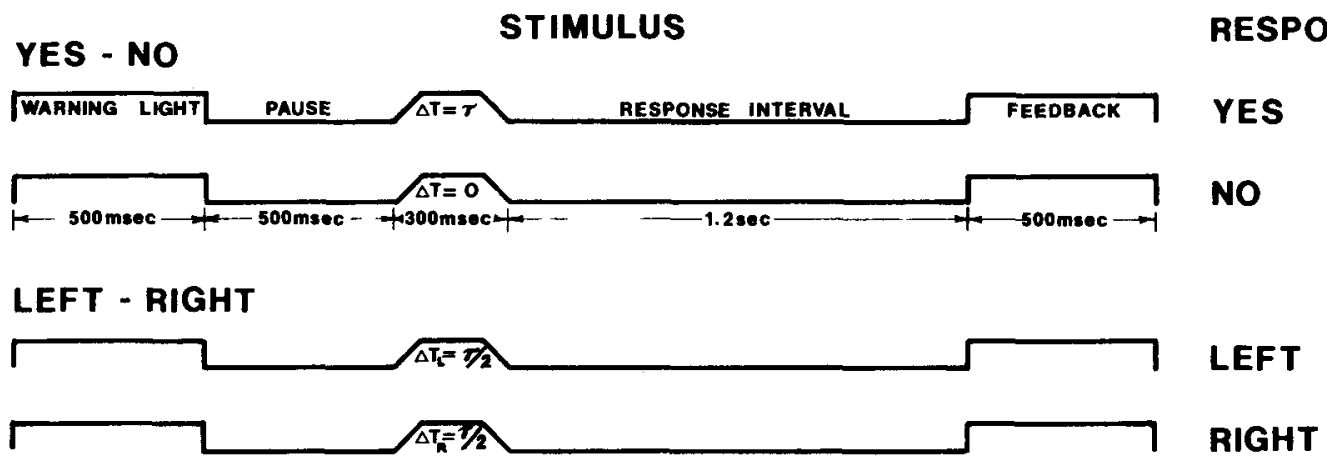

\section{SAME - DIFFERENT}

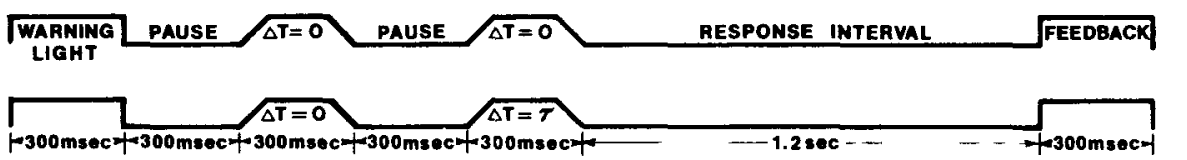

SAME

DIFFERENT

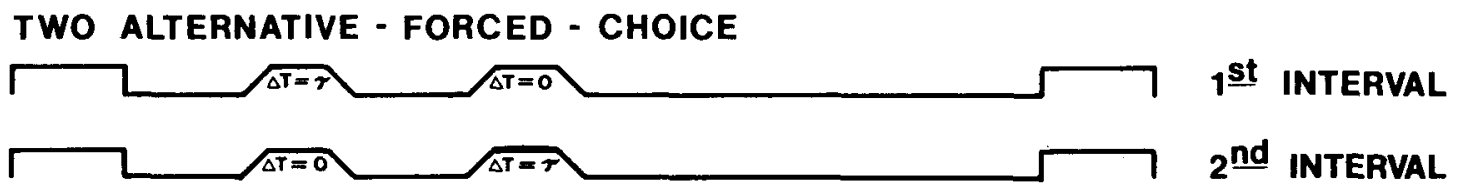

Fig. 1. The timing diagrams for each of the four psychophysical procedures are shown. The stimulus conditions are shown on the left and the appropriate responses on the right. Each task consists of a warning interval, an observation interval, a response interval, and a feedback interval. $A$ value of the interaural temporal difference $(\Delta T)$ is presented in each observation interval. $\Delta T$ is either $\tau$ or 0 in the yes-no task. $\tau / 2$ is added either to the left ear $\left(\Delta T_{L}\right)$ or to the right ear $\left(\Delta T_{R}\right)$ in the left-right task. Two values of $\Delta T(0$, or $\tau)$ are presented in the two interval tasks (same-different and two-alternative forced-choice tasks).

The two Os were 22-year-old University of Florida coeds with normal hearing. They were given at least 1,000 practice trials in each of the four procedures $(4,000$ total practice trials) before data collection was begun. The Os were instructed as to the type of task being employed and how to use the feedback lights to determine the correct response. That is, no direct instructions were given concerning what the 0 "should" listen for in each procedure.

\section{RESULTS AND DISCUSSION}

The data from the two Os are plotted in Fig. 2 as $\mathrm{P}(\mathrm{C})_{2}$ vs $\Delta \mathrm{T}$. The four curves represent the performance obtained in each of the four procedures. $\mathrm{P}(\mathrm{C})_{2}$ was computed by obtaining the probabilities of a hit and correct rejection in each task. These two probabilities were then summed and divided by two (McFadden, 1970). The data points plotted in Fig. 2 represent the mean $\mathrm{P}(\mathrm{C})_{2}$ computed over the 500 trials at both values of $\Delta \mathrm{T}$. McFadden (1970) showed that $\mathrm{P}(\mathrm{C})_{2}$ can pe affected by response bias and by the bias in obtained stimulus proportions. In the present study, both the response bias and the obtained stimulus proportions were between .45 and .55 (computed every 100 trials). Thus, according to McFadden's results, there will be little error associated with comparing the four tasks in terms of $\mathrm{P}(\mathrm{C})_{2}$. In addition, the variance in $\mathrm{P}(\mathrm{C})_{2}$ obtained over the 500 trials for each value of $\Delta \mathrm{T}$ was never greater than the Bournoulli variability obtained by assuming that the mean $\mathrm{P}(\mathrm{C})_{2}$ for each value of $\Delta \mathrm{T}$ equalled the binomial probability (Green \& Swets, Appendix III, 1966). Finally, the slopes of the four psychometric functions for each $\mathrm{O}$ are approximately parallel. Thus, the four psychometric functions for each $O$ represent an accurate estimate of the performance in each of the four tasks. This allows for accurate comparisons among the four procedures.

Figure 3 is a plot of the four psychometric functions plotted as $\mathrm{d}^{\prime}$ vs $\Delta T$. In this figure, $\mathrm{d}^{\prime}$ for each task was obtained from the tables of Swets (1964). The values of $\mathrm{d}^{\prime}$ for the Y-N, L-R, and S-D tasks were obtained by entering the respective hits and false alarms in the yes-no $d^{\prime}$ table. The $d^{\prime}$ values for the $2 A F C$ task were obtained by using $\mathrm{P}(\mathrm{C})_{2}$ and the two alternative forced-choice table in Swets (1964).

According to TSD (see Green \& Swets, 1966; and Sorkin, 1964), this is the proper procedure for estimating $\mathrm{d}^{\prime}$ and TSD predicts that these four estimates for each value of $\Delta T$ should yield the same value of $\mathrm{d}^{\prime}$. In order to demonstrate that this prediction was not verified in the present study, the Y-N psychometric function is drawn in Fig. 3. Also, the range of $d^{\prime}$ values obtained over the 500 trials for each value of $\Delta T$ in the 
Fig. 2. $\mathrm{P}(\mathrm{C})_{2}$ is plotted as a function of $\Delta T$ for each $O$. The four tasks, yes-no $(0-0)$, left-right ( $\square-\square)$, two-alternative forced-choice $(X-X)$, and same-different $(\triangle-\Delta)$, are shown. The two figures are for the two $\mathrm{Os}(\mathrm{O}) \mathrm{and}$ $O$ 11). See text for definition of $P(C)_{2}$.

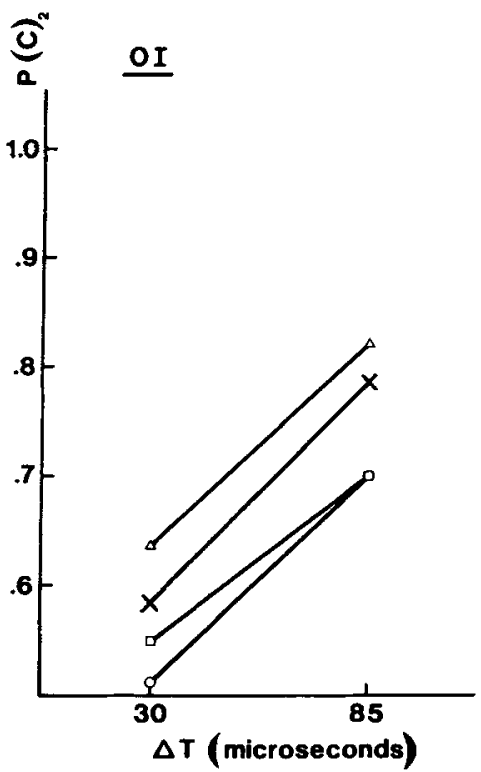

Y.N tasks is shown in Fig. 3 as the vertical bars. As can be seen, the mean values of $d^{\prime}$ from the $2 A F C$ and $L-R$ tasks lie within the range of values obtained in the Y-N tasks. However, the mean values of $d^{\prime}$ obtained in the S-D task lie outside this range, indicating that the S-D task is significantly different from the Y-N, 2AFC, or L-R tasks.

In Fig. 4, the four psychometric functions are again plotted as d' vs $\Delta T$. The plots for the Y-N, L-R, and S-D tasks are the same as in Fig. 3. The $\mathrm{d}^{\prime}$ values for the 2AFC task were obtained by using the hits and false alarms computed for this condition and the yes-no $\mathrm{d}^{\prime}$ table. That is, the data points in the 2AFC tasks shown in Fig. 4 are displaced up by the $\sqrt{2}$ from those plotted in Fig. 3. Psychometric functions are drawn through the

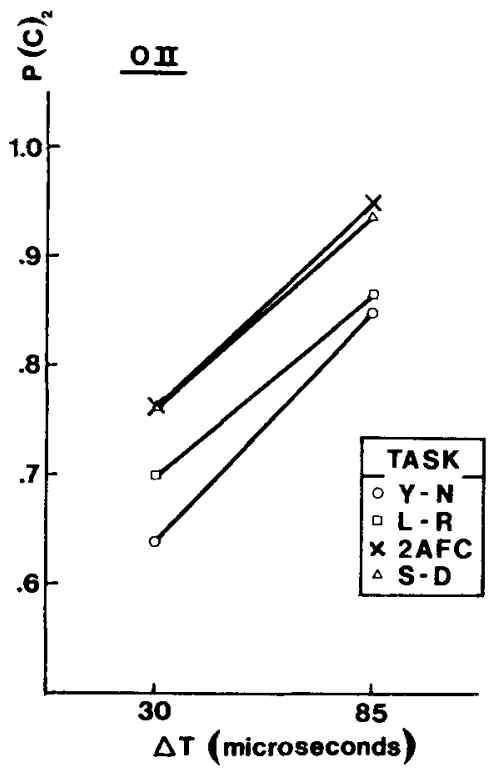

Y-N and S-D conditions. The vertical bars represent the range of the values of $\mathrm{d}^{\prime}$ obtained in the Y.N and S-D procedures. Figure 4 indicates that there are two groupings of data: the similar values of $d$ ' produced by the Y-N and the L-R tasks and those produced by the $2 \mathrm{AFC}$ and $\mathrm{S}-\mathrm{D}$ tasks.

Figure 3 demonstrates that the Os are far more sensitive in the S.D task than would be predicted by TSD. This prediction was made on the assumption that the Os were deciding between two stimulus conditions, i.e., two values of $\Delta T$. Since changes in $\Delta T$ result in different locations of an image in lateral space, the Os' decisions were assumed to be between two positions in lateral space. For instance, in the $\mathrm{Y}-\mathrm{N}$ task, the $\mathrm{O}$ was deciding between an image located near midline (no
Fig. 3. $d^{\prime}$ is plotted as a function of $\Delta T$ for each 0 . The data points are for the same four conditions described in Fig. 2. The curve is drawn through the data of the yes-no task. The vertical bars represent the range of the values of $d^{\prime}$ obtained in the yes-no task. Data from two Os are shown (O 1 and 0 11). See text for description of d'.
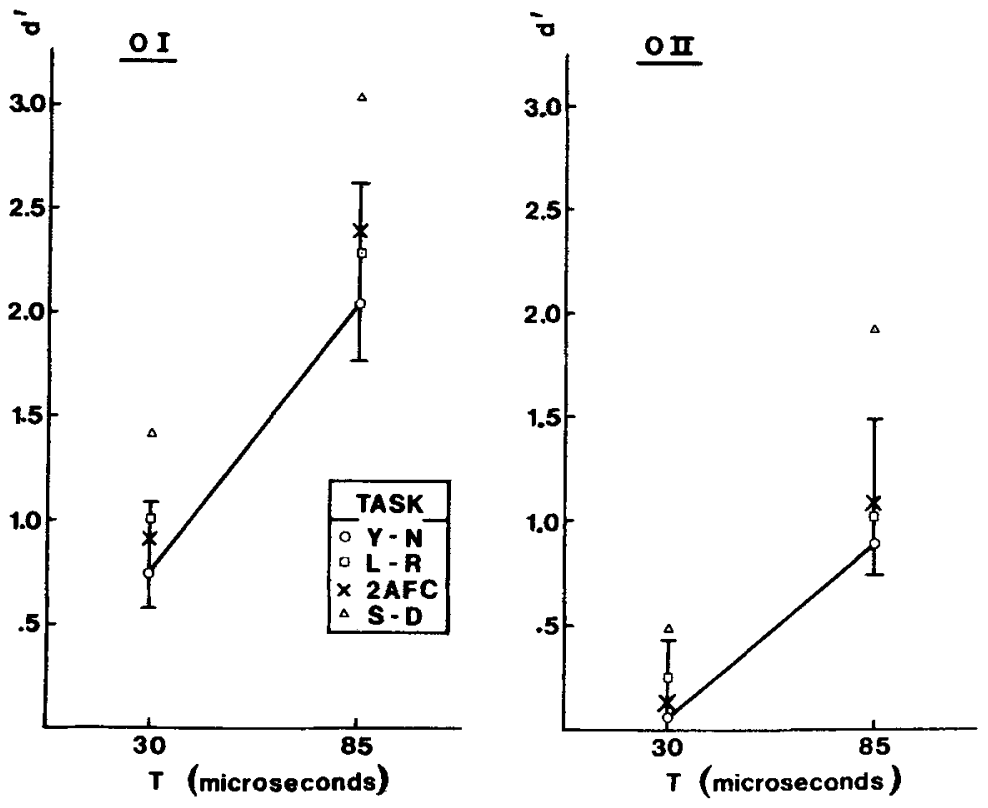

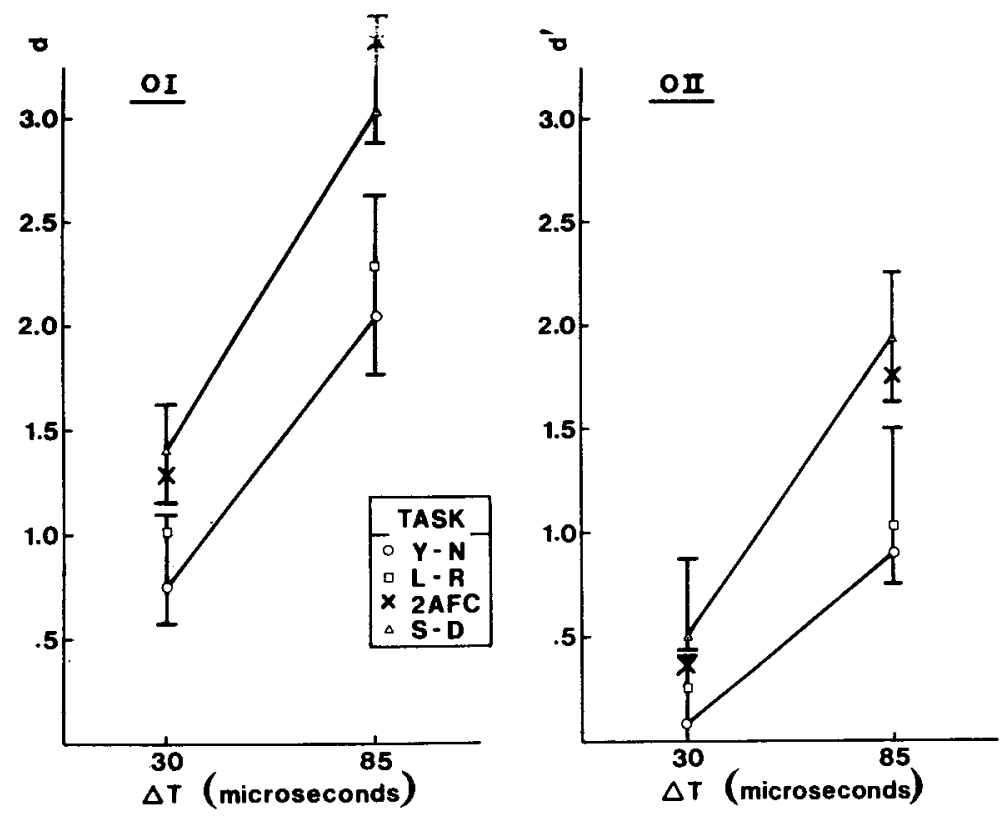

Fig. 4. $d^{\prime}$ is plotted as a function of $\Delta T$ for each 0 . The yes-no, same-different, and left-right data points, as well as the curve through the yes-no data and the vertical range bars for the yes-no task, are the same as in Fig. 3. The data for the two-alternative forced-choice task are displaced up by the $\sqrt{2}$ from those shown in Fig. 3. A curve is drawn through the same-different data, and the verticle bars through the same-different data represent the range of $d^{\prime}$ values obtained in this task. See text for further information and definition of $d^{\prime}$.

interaural delay) and one located toward the right ear (interaural delay).

Os in the S-D and 2AFC tasks report that they were not aware of two images (one for each interval) in each task, but rather that of movement of the image. In the S-D task, the image either moved to the right (a different trial) or it did not move (a same trial). In the 2AFC task, the image moved either to the right (a second-interval trial) or to the left (a first-interval trial). Thus, the two-interval tasks did not consist of two events, as was assumed, but of one event: lateral movement.

This indicates that the S-D and $2 \mathrm{AFC}$ tasks, although physically two-interval tasks, may be considered formally as single-interval tasks in which movement is the cue. The single-interval Y-N and L-R tasks may be thought of as single-interval tasks in which location of the lateral image is the cue. The data in Fig. 4 were plotted using this assumption. Both Fig. 4 and Fig. 2 demonstrate that there are two groupings of the data. That is, performance in the 2AFC and S-D tasks is similar, as is performance in the Y-N and L-R tasks.

Although TSD can be used to demonstrate that the Y-N and L-R tasks are similar and that the 2AFC and S-D are similar, it cannot account for the difference between the two types of tasks. In order to predict this difference, a model is required which predicts that Os are more sensitive to lateral motion (change in lateral position) than to lateral position.

It has been assumed that both the $2 \mathrm{AFC}$ and S-D tasks produce the lateral motion cue which the Os use in these procedures. The values of $\mathrm{d}^{\prime}$ obtained in the $2 \mathrm{AFC}$ task were similar to those obtained in the two single-interval tasks (Fig. 3). One might assume, therefore, that lateral position is the cue for the L-R, Y-N, and 2AFC tasks and that lateral motion is the cue in the S-D task, since only the S-D task produced the disparate values of $d^{\prime}$. However, since (1) the Os reported motion in both the $2 \mathrm{AFC}$ and S-D tasks, (2) the values of $d^{\prime}$ were similar for the $2 A F C$ and $S-D$ conditions, as plotted in Fig. 4, and (3) these two tasks are similar paradigms, it is assumed that in both the 2AFC and S-D tasks the $\mathrm{O}$ used lateral motion as the cue for detection.

The observation that $O$ s are more sensitive in lateralization tasks containing a standard or a comparison stimulus is not new (Zwislocki \& Feldman, 1966). The data of the present study suggest that the standard stimulus when used in two-interval tasks helps in establishing lateral motion. Os may be more sensitive to motion than to position of the lateral image.

The data from the two-interval tasks (S-D and 2AFC) are similar to those obtained by other investigators. The value of $\Delta \mathrm{T}$ estimated at a $\mathrm{P}(\mathrm{C})_{2}$ equal to $75 \%$ averages 27 microsec for $\mathrm{O} 1$ and $55 \mathrm{microsec}$ for $\mathrm{O} 11$. These values compare well with those obtained by Zwislocki and Feldman (1966, 30 microsec), Klumpp and Eady (1966, 27 microsec), Mills (1958, 35 microsec), and Yost (in press, 35 microsec). The slopes of the psychometric functions in the present investigation are also similar to those obtained in these experiments.

In conclusion, the data of the present study suggest that Os process stimuli differently in lateralization tasks than in similar tasks which involve masking. That is, in masking experiments, the $O$ appears to use the same cue for detection in both single- and two-interval experiments. However, in lateralization experiments, the two-interval procedures appear to provide a different cue for differentiation than the single-interval tasks. In other words, lateral position is the cue in single-interval tasks and lateral motion is the cue in two-interval tasks. 


\section{REFERENCES}

Green, D. M., \& Swets, J. A. Signal detection theory and psychoacoustics. New York: Wiley, 1966.

Klumpp, R. G.. \& Eady, E. R. Some measurements of interaural time difference thresholds. Journal of the Acoustical Society of America, 1956, 28, 859-860.

McFadden, D. Three computational versions of proportion correct for use in forced-choice experiments. Perception \& Psychophysics, $1970,8,336-343$.

Mills, A. W. On the minimum audible angle, Journal of the Acoustical Society of America, 1958, 30, 237-246.

Robinson, D. E., \& Watson, C. S. Psychophysical methods in modern psychoacoustics. In J. V. Tobias (Ed.), Foundations of modern auditory theory. Vol. II. New York: Academic Press, 1972 .

Sorkin, R. D. Extension of the theory of signal detectability to matching procedures in psychoacoustics. In J. A. Swets (Ed.), Signal detection and recognition by human observers. New York: Wiley, 1964 .
Swets, J. A. Signal detection and recognition by human observers. New York: Wiley. 1964.

Yost, W. A. Interaural phase-discriminations. Journal of the Acoustical Society of America, in press.

Zwislocki, J., \& Feldman, R. S. Just noticeable differences in dichotic phase. Journal of the Acoustical Society of America, $1956,28,860-864$.

\section{NOTE}

1. In the left-right task, the difference in the interaural temporal difference between the two types of presentations was kept equal to $\tau$, which is the same as the difference in the yes-no task. That is, in the left-right task, the total difference in interaural time that the $O$ is to detect is the same as in the yes-no task $(\tau)$.

(Accepted for publication December 26, 1973.) 